\title{
Factors associated with stillbirths among women delivering at a resource limited tertiary hospital in Malawi
}

\begin{abstract}
Background: Stillbirths remain a major public health issue worldwide with an estimated 3 million deaths per year globally. We investigated the factors associated with stillbirths in fetuses of at least 28 weeks' gestation or 1000 grams at birth.

Methods: We performed a hospital-based, cross-sectional study among women who delivered stillbirths at Kamuzu Central Hospital $(\mathrm{KCH})$ in Lilongwe, Malawi from MayNovember 2017. Eligible women were enrolled after obtaining informed consent, and their demographic and reproductive health information was collected. Blood samples were collected for full blood count, malaria, blood glucose, syphilis, and HIV testing, and the probable risk factors associated with stillbirths were assessed.
\end{abstract}

Results: A total of 1,687 deliveries with 126 stillbirths occurred during the 6-month period, representing a stillbirth rate of 79 per 1,000 births. Seventy percent of these stillbirths were diagnosed on admission at $\mathrm{KCH}$, and about $49 \%$ were fresh stillbirths. Half of the stillbirths had a birthweight of at least 2,500g, and the majority of these stillbirths were fresh $(60 \%)$. The following factors were associated with stillbirth: uterine rupture (15.1\%), placental abruption (14.3\%), Hypertension (10.3\%), obstructed/prolonged labor (5.8\%), syphilis (7.1\%), malaria (2.4\%), congenital anomalies $(2.4 \%)$, and diabetes $(1.5 \%)$.

Conclusions: The stillbirth rate at $\mathrm{KCH}$ is high, and most fetal deaths occurred prior to arrival at $\mathrm{KCH}$. Although most of the stillbirths were unexplained, uterine rupture and abruption placenta emerged as major factors associated with stillbirths and these are largely preventable even in resource limited settings.

Keywords: stillbirths, adverse birth outcomes, uterine rupture, hypertensive disorders of pregnancy, cesarean section
Volume 12 Issue 5 - 202I

\author{
Friday Saidi, ${ }^{1,2}$ Grace Chiudzu, ${ }^{2}$ Maganizo \\ Chagomerana,' Beteniko Milala,' Jennifer H \\ Tang ${ }^{1,3}$ \\ 'University of North Carolina Project-Malawi (UNCPM), \\ Lilongwe, Malawi \\ ${ }^{2}$ Department of Obstetrics and Gynecology, Kamuzu Central \\ Hospital, Malawi Ministry of Health, Lilongwe, Malawi \\ ${ }^{3}$ Department of Obstetrics and Gynecology, University of \\ North Carolina, Chapel Hill, NC, USA
}

Correspondence: Friday Saidi, UNC Project Lilongwe Private Bag A-I 04 Lilongwe, Department of Obstetrics and Gynecology, Kamuzu Central Hospital, Malawi Ministry of Health, Malawi, Tel 00265995403 I I3,Email fsaidi@unclilongwe.org

Received: August 05, 2021 | Published:September 16, 2021

\section{Background}

Approximately 3 million stillbirths occur yearly worldwide, representing an estimated global stillbirth rate of about 18.4 per 1,000 births ( $\geq 28$ completed weeks' gestation). ${ }^{1}$ The majority of these stillbirths (nearly $98 \%$ ) occur in low- and middle-income countries. ${ }^{2}$ In Malawi, the stillbirth rate is estimated at 21.8 per 1,000 total births; higher compared to other resource-limited countries, and much higher than in high-income countries. ${ }^{3}$ Stillbirth can be a source of significant grief and may lead to long-term psychological issues for the mother and her family. ${ }^{4-6}$ In 2014, the World Health Organisation (WHO) released an action plan aimed at reducing the stillbirth rate to 12 or lesser per 1,000 births by 2030 in every country; however, the progress has been slow. ${ }^{7,8}$

Stillbirths remain an enormous challenge in the clinical care of pregnant women. In low-income countries, the etiology of stillbirths is difficult to assess, and the causes of stillbirth in most cases are unclear or unexplained. ${ }^{8}$ Currently in Malawi, the causes of stillbirth are determined by verbal autopsy - gathering health information through interviews and discussions to determine the possible cause of death. ${ }^{9}$ Although approximately $25-60 \%$ of stillbirths are not associated with any obvious risk factors or causes, various risk factors and maternal conditions have been described. ${ }^{10-12}$ Most of the known conditions that cause stillbirth can be diagnosed and treated.
Describing the exact timing and factors associated with stillbirths can indicate the quality of care of an institution through detection of specific shortcomings in the obstetric care. ${ }^{9}$ Therefore, we conducted this study to:

1) Assess the demographic and reproductive health characteristics of women who experience stillbirth at Kamuzu Central Hospital $(\mathrm{KCH})$

2) Describe the medical conditions and risk factors associated with stillbirths amongst women delivering at $\mathrm{KCH}$

3) Determine the stillbirth rate for women who delivered at or were referred to this tertiary facility.

\section{Methods}

\section{Study setting and design}

We conducted a cross-sectional study of women who experienced a stillbirth at KCH from May to November 2017. KCH is a referral hospital located in Lilongwe, the capital of Malawi with an estimated population of about 1.2 million people. ${ }^{13}$ This study was conducted at KCH's Ethel Mutharika Maternity Ward (EMMW). EMMW is staffed by the Department of Obstetrics and Gynecology at KCH and has 220 beds. A significant proportion of patients at EMMW are referred from 
hospitals and health centers in its 9 referral districts. The study was approved by the University of Malawi College of Medicine Research Committee (COMREC). Written, informed consent was obtained from each mother before data collection.

We based our definition of stillbirth on the World Health Organization (WHO) international definition of viability. Therefore, stillbirth was defined as death in the uterus of an infant at $\geq 28$ weeks of gestation or having a birthweight $\geq 1,000 \mathrm{~g} .{ }^{14} \mathrm{~A}$ fresh stillbirth was defined as the intrauterine death of a fetus during labor or delivery, while a macerated stillbirth was defined as an intrauterine death of a fetus occurring before the onset of labor and the fetus shows degenerative change. ${ }^{15}$ Gestational age was determined by last menstrual period, fundal height estimation, or ultrasound.

We included women who delivered a stillborn with Apgar of 0 at 1 and 5 minutes. Women with multiple gestation, i.e. twins pregnancy were included only if the first-born was a stillborn. We excluded women who could not give consent, for example, critically ill patients in the High Dependency Unit or Intensive Care Unit, psychiatric patients, women who delivered outside of KCH EMMW, and women who declined participation in the study.

Participants were recruited from the EMMW labor ward or postnatal wards. The study Principal Investigator (PI) or trained labor ward and postnatal nurse performed the screening using an eligibility checklist and obtained informed consent from women 18 years and above, and pediatric assent and parental consent for those below 18 years of age. All study specific procedures were performed only after informed consent was obtained.

The study procedures involved a retrospective review of maternity records and blood sample collection for full blood count (FBC), random blood sugar (RBS), syphilis rapid plasma reagin (RPR), malaria rapid diagnostic tests (MRDT) and HIV testing (for HIV negative or unknown if HIV testing was not done at within 3 months of delivery of the stillbirth). For all syphilis RPR positive tests, a confirmatory syphilis Treponema pallidum particle agglutination assay (TPPA) was performed on the same sample.
Data collection was performed using a structured questionnaire designed to collect information on socio-demographic characteristics, antenatal care attendance, medical history, pregnancy outcomes, and probable cause of stillbirth. Laboratory data were transcribed onto a study specific laboratory form.

Data was entered into a Microsoft Access database and analyzed using Stata statistical package (Version 15.0, Stata Corp LLC, College Station, Texas, USA). We summarized participants' characteristics using medians (continuous variables) and percentages (categorical variables) and compared the characteristics between participants that delivered a fresh stillbirth to those that delivered a macerated stillbirth using Pearson's Chi-squared and Fisher's exact tests.

\section{Results}

From 08 May 2017 to 09 November 2017, there were 1,687 deliveries at KCH EMMW, 1554 livebirths and 133 stillbirths. Of the 133 women who delivered stillbirths, $126(95 \%)$ women were enrolled in the study. Seven participants were not enrolled because the study team missed them or did not meet eligibility criteria. The overall stillbirth rate at EMMW during this period was 79 stillbirths per 1,000 births (including the seven women who were not enrolled).

Among the participants that were enrolled, 95 (75\%) had intrauterine fetal demise (IUFD) diagnosed on admission and 31 $(25 \%)$ had fetal heart activity on admission to $\mathrm{KCH}$. Therefore, the in-hospital stillbirth rate in our study was 18 per 1,000 births. Of the 126 stillbirths, 61 (49\%) were fresh stillbirths. Of the in-hospital stillbirths, most were fresh stillbirths and were attributed to delays between making the decision to deliver and the actual time they were delivered, with an average delay time of around 1 hour.

The median age of the women was 27 years range: 16 - 43 years. The majority of the women were aged between 20 and 34 years $(75.3 \%)$, had singleton pregnancies $(92.1 \%)$, were married $(91.9 \%)$, were multiparous $(73.7 \%)$, and had some or had completed primary education $(59.8 \%$ ) (Table 1). About $8 \%$ of the women had a history of prior stillbirth and more than half of the women (51.5\%) had an unknown baseline antenatal hemoglobin.

Table I Demographic and reproductive characteristics of women with stillbirth

\begin{tabular}{|c|c|c|c|c|c|c|c|}
\hline \multirow{2}{*}{ Characteristic } & \multicolumn{2}{|c|}{ Total $(\mathrm{N}=126)$} & \multicolumn{2}{|c|}{ FSB $(n=61)$} & \multicolumn{2}{|c|}{ MSB $(n=65)$} & \multirow{2}{*}{ p-value } \\
\hline & $\mathbf{N}$ & $\%$ & $\mathbf{N}$ & $\%$ & $\mathbf{N}$ & $\%$ & \\
\hline \multicolumn{7}{|c|}{ Maternal Age (Median 27 years) } & 0.06 \\
\hline $12-19 \mathrm{yrs}$ & 16 & 12.7 & 8 & 13.1 & 8 & 12.3 & \\
\hline $20-34$ yrs. & 95 & 75.3 & 50 & 82 & 45 & 69.2 & \\
\hline$>35$ yrs. & 15 & 12 & 3 & 4.9 & 8 & 18.5 & \\
\hline Educational level & & & & & & & 0.46 \\
\hline None & 11 & 8.7 & 7 & 11.4 & 4 & 6.2 & \\
\hline Primary & 75 & 59.8 & 37 & 60.7 & 38 & 58.5 & \\
\hline Secondary/Tertiary & 40 & 31.8 & 17 & 27.9 & 23 & 35.4 & \\
\hline Marital Status & & & & & & & 0.2 \\
\hline Married & 114 & 91.9 & 56 & 94.9 & 54 & 89.2 & \\
\hline Other & 10 & 2.4 & 3 & 5.1 & 7 & 10.8 & \\
\hline
\end{tabular}




\begin{tabular}{|c|c|c|c|c|c|c|c|}
\hline \multirow{2}{*}{ Characteristic } & \multicolumn{2}{|c|}{ Total $(N=126)$} & \multicolumn{2}{|c|}{ FSB $(n=6 I)$} & \multicolumn{2}{|c|}{ MSB $(n=65)$} & \multirow{2}{*}{ p-value } \\
\hline & $\mathbf{N}$ & $\%$ & $\mathbf{N}$ & $\%$ & $\mathbf{N}$ & $\%$ & \\
\hline Parity & & & & & & & 0.88 \\
\hline Nulliparous & 38 & 26.3 & 18 & 29.5 & 20 & 30.8 & \\
\hline Multiparous & 88 & 73.7 & 43 & 70.5 & 45 & 69.2 & \\
\hline Prior Stillbirth & & & & & & & 0.58 \\
\hline Yes & 10 & 7.9 & 6 & 9.8 & 4 & 6.2 & \\
\hline No & 116 & 92.1 & 55 & 90.2 & 61 & 93.8 & \\
\hline Height & & & & & & & 0.47 \\
\hline$<150 \mathrm{~cm}$ & 14 & 11.2 & 5 & 8.2 & 9 & 14.1 & \\
\hline$>150 \mathrm{~cm}$ & 111 & 88 & 55 & 91.8 & 56 & 85.9 & \\
\hline ANCVisits & & & & & & & 0.31 \\
\hline$<=1$ & 44 & 34.9 & 24 & 39.3 & 20 & 30.8 & \\
\hline $2-5$ & 82 & 65 & 37 & 60.7 & 45 & 69.2 & \\
\hline Twin pregnancy & & & & & & & 0.45 \\
\hline No & 116 & 92.1 & 55 & 90.2 & 61 & 93.9 & \\
\hline Yes & 10 & 7.9 & 6 & 9.8 & 4 & 6.1 & \\
\hline \multicolumn{3}{|c|}{ Baseline Haemoglobin $(\mathrm{Hb})$} & & & & & 0.64 \\
\hline$>\mid \lg / \mathrm{dl}$ & 25 & 20 & 2 & 3.3 & 4 & 6.2 & \\
\hline $6-10.9 \mathrm{~g} / \mathrm{dl}$ & 30 & 23.8 & 16 & 26.3 & 14 & 21.5 & \\
\hline$<6 \mathrm{~g} / \mathrm{dl}$ & 6 & 4.8 & 14 & 23 & II & 16.9 & \\
\hline Unknown & 65 & 51.5 & 29 & 47.5 & 36 & 55.4 & \\
\hline Mode of Delivery & & & & & & & $0.0004^{*}$ \\
\hline SVD & 60 & 48 & 20 & 32.8 & 40 & 62.5 & \\
\hline Breech & 19 & 15.2 & 13 & 21.3 & 6 & 9.4 & \\
\hline Vacuum & 2 & 1.6 & 2 & 3.3 & 0 & 0 & \\
\hline Cesarean & 44 & 35.2 & 26 & 41.6 & 18 & 28.1 & \\
\hline
\end{tabular}

$*_{p}<0.05$. Some values do not add up to $100 \%$ due to missing data

Women with a macerated stillbirth were more likely to be $>35$ years $(n=8,18.5 \%)$ than women with a fresh stillbirth $(n=3,4.9 \%), p=0.06$. The overall cesarean section rate was $35.2 \%$ and was significantly higher among women with a fresh stillbirth $(n=26,41.6 \%)$ than among women with a macerated stillbirth $(n=18,28.1 \%), p=0.004$. The average time taken between making the decision to perform a cesarean section to the time of extracting the baby was around 1 hour and was irrespective of the indication of the cesarean section.

Exactly half of the stillbirths had a birth weight of at least 2,500 g, and $37(29.3 \%)$ of these 63 stillbirths were fresh. Fresh stillbirths were more likely to weigh 2,500 g or more $(60.7 \%)$ when compared to macerated stillbirths $(40.6 \%), p=0.08$. The majority of the stillbirths $(\mathrm{n}=74,58.8 \%)$ were preterm $(<37$ weeks gestation), but $52(41.2 \%)$ occurred at term ( $\geq 37$ weeks gestation). There was a statistically significant difference between the type of stillbirth and presence of fetal heart activity, with more macerated stillbirths $(84.6 \%)$ than fresh stillbirths $(65.6 \%)$ having no fetal heart activity on admission $(\mathrm{p}=0.02)$ (Table 2).

The prevalence of HIV amongst the women with stillbirths in this study was $10.3 \%$, and $49.2 \%$ had anemia with hemoglobin $<11 \mathrm{~g} /$ dl. All of the HIV-infected women were already on antiretroviral medication at time of enrolment. There were no new HIV diagnoses made amongst women who had initial HIV negative results during their antenatal care (Table 3 ). 
Table 2 Fetal Characteristics of the stillbirths

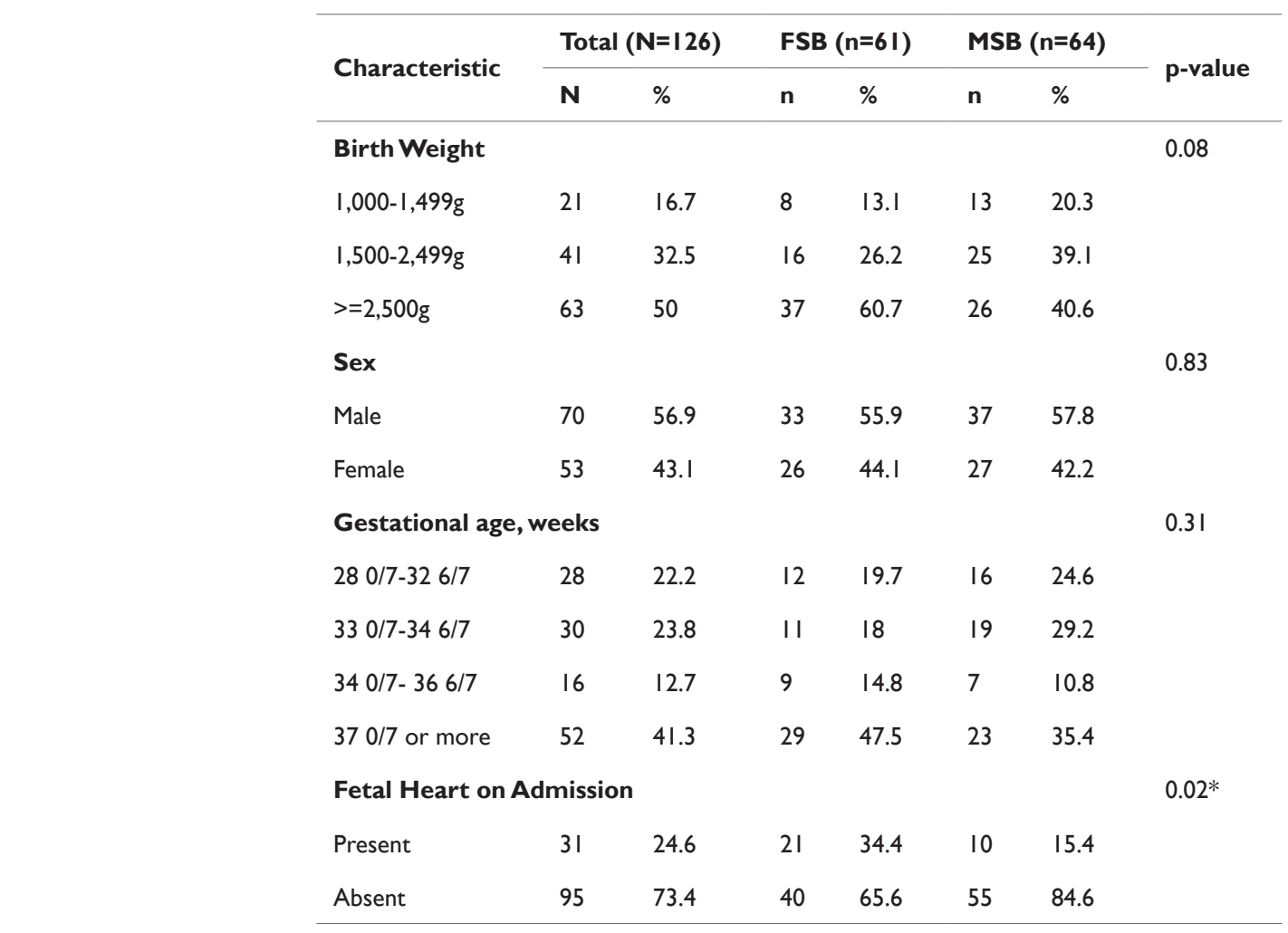

$* \mathrm{p}<0.05$

Table 3 Maternal medical conditions associated with the stillbirths

\begin{tabular}{|c|c|c|c|c|c|c|c|}
\hline \multirow{2}{*}{ Characteristic } & \multicolumn{2}{|c|}{ Total $(\mathbf{N}=\mid 26)$} & \multicolumn{2}{|c|}{ FSB $(\mathbf{N}=6 I)$} & \multicolumn{2}{|c|}{ MSB (N=64) } & \multirow{2}{*}{ p-value } \\
\hline & $\mathbf{N}$ & $\%$ & $\mathbf{N}$ & $\%$ & $\mathbf{N}$ & $\%$ & \\
\hline HIV & & & & & & & 0.56 \\
\hline Negative & 113 & 89.7 & 56 & 91.8 & 57 & 87.7 & \\
\hline Positive & 13 & 10.3 & 5 & 8.2 & 8 & 12.3 & \\
\hline Malaria & & & & & & & 0.6 \\
\hline Negative & 123 & 97.6 & 59 & 96.7 & 64 & 98.5 & \\
\hline Positive & 3 & 2.4 & 2 & 3.3 & I & 1.5 & \\
\hline Hypertension & & & & & & & 0.39 \\
\hline Yes & 13 & 10.3 & 8 & I3.I & 5 & 7.7 & \\
\hline No & 113 & 89.7 & 53 & 86.9 & 60 & 92.3 & \\
\hline \multicolumn{3}{|c|}{ Anemia, Mean Hb I $0.3 \pm 2.9$} & & & & & 0.22 \\
\hline None & 57 & 45.2 & 24 & 39.3 & 33 & 50.8 & \\
\hline Yes & 69 & 54.8 & 37 & 60.7 & 32 & 49.2 & \\
\hline Syphilis & & & & & & & 0.49 \\
\hline Negative & 117 & 92.9 & 58 & 95.1 & 59 & 90.8 & \\
\hline Positive & 9 & 7.1 & 3 & 4.9 & 6 & 9.2 & \\
\hline Diabetes & & & & & & & 0.49 \\
\hline Yes & 1 & 0.8 & 0 & 0 & I & 1.5 & \\
\hline No & 125 & 99.2 & 61 & 100 & 64 & 98.5 & \\
\hline
\end{tabular}

Citation: Saidi F, Chiudzu G, Chagomerana M, et al. Factors associated with stillbirths among women delivering at a resource limited tertiary hospital in Malawi. Obstet Gynecol Int J. 202I;I2(5):289-295. DOI: I0.I5406/ogij.202I.I2.00594 
We had newly diagnosed syphilis $(\mathrm{n}=9,7.1 \%)$, malaria $(\mathrm{n}=3$, $2.4 \%)$ and diabetes $(n=1,1.5 \%)$. All the women who tested positive for syphilis using the syphilis RPR tests had confirmed positive results on syphilis TPPA tests, and all had high titers of at least 1:4. None of the women who had positive syphilis result had had a prior baseline VDRL test during their antenatal care visits. None of the maternal medical conditions we diagnosed was found to be significantly associated with type of stillbirth.

For the antepartum factors associated with stillbirths, $14.3 \%$ had placental abruption as the probable cause of fetal demise, and there was no difference between fresh and macerated stillbirths. Hypertensive disorders accounted for $10.3 \%$ of stillbirths, again with no difference between fresh and macerated stillbirths. Congenital anomalies were present in only $2.4 \%(n=3)$ of the stillbirths, though fetal autopsy could not be done in this study, there could have been some cases that were missed.

For the intrapartum factors, birth asphyxia accounted for $8.7 \%$ of the probable causes of the stillbirths. Cord accidents accounted for $5.6 \%$ of stillbirths, and prolonged labor for $5.8 \%$ of stillbirths, all of which occurred in fresh stillbirths.

Uterine rupture resulted in $15.0 \%$ of the stillbirths, and the proportion of uterine rupture was $21.3 \%$ among fresh stillbirths and $9.2 \%$ among macerated stillbirths $(\mathrm{p}=0.05)$. Of the 19 women who had uterine rupture, $18(94.7 \%)$ were among women with one prior cesarean section who were undergoing vaginal birth after cesarean section (VBAC) at different health facilities. Two of the 19 patients $(15.1 \%)$ had their uterine rupture occur within EMMW labor ward whilst attempting VBAC (Table 4).

Table 4 Probable causes of Stillbirths

\begin{tabular}{|c|c|c|c|c|c|c|c|}
\hline \multirow{2}{*}{ Characteristics } & \multicolumn{2}{|c|}{ TOTAL (N= I 26) } & \multicolumn{2}{|c|}{ FSB $(n=61)$} & \multicolumn{2}{|c|}{ MSB $(n=64)$} & \multirow{2}{*}{ p-value } \\
\hline & $\mathbf{N}$ & $\%$ & $\mathbf{n}$ & $\%$ & $n$ & $\%$ & \\
\hline \multicolumn{8}{|l|}{ Antepartum factors } \\
\hline Placenta Abruption & 18 & 14.3 & 7 & 11.5 & II & 17 & 0.38 \\
\hline Hypertensive disease & 13 & 10.3 & 8 & 13.1 & 5 & 7.7 & 0.39 \\
\hline Maternal Infection & 10 & 7.4 & 4 & 6.6 & 6 & 9.2 & 0.75 \\
\hline Diabetes Mellitus & I & 0.8 & 0 & 0 & I & 1.6 & 0.48 \\
\hline Congenital anomalies & 3 & 2.4 & I & 1.6 & 2 & 3.1 & 0.52 \\
\hline \multicolumn{8}{|l|}{ Intrapartum factors } \\
\hline Asphyxia & 11 & 8.7 & 8 & 13.1 & 3 & 4.6 & 0.08 \\
\hline Cord Accidents & 7 & 5.6 & 3 & 4.9 & 4 & 6.1 & 0.76 \\
\hline Uterine Rupture & 19 & 15.1 & 13 & 21.3 & 6 & 9.2 & 0.05 \\
\hline Obstructed labor & 6 & 5.8 & 6 & 9.8 & 0 & 0 & 0.07 \\
\hline Unexplained & 31 & 24.6 & 9 & 14.8 & 22 & 33.9 & $0.01 *$ \\
\hline
\end{tabular}

$*_{p}<0.05$

The most common cause of the stillbirths (24.6\%), however, was unexplained. Macerated stillbirths were more likely to be unexplained $(33.9 \%)$ than fresh stillbirths $(14.8 \%), p=0.01$. The frequency of unexplained stillbirth seemed to increase with increasing gestational age, with a majority of these stillbirths $(n=13)$ noted between 37 to 40 weeks.

\section{Discussion}

Stillbirth is still a challenge in many development countries including Malawi. In our study at a referral hospital in Malawi, the stillbirth rate was more than double the reported national stillbirth rate as well as the reported Sub-Saharan Africa stillbirth rate (32 per 1,000 births). ${ }^{16}$ The cause of stillbirth was unexplained for a quarter of our cases, and the most known causes were uterine rupture and placental abruption. Most of the causes of these stillbirths (uterine rupture, placenta abruption) were potentially preventable or treatable, or could have interventions developed. Of note, only a quarter of women with stillbirth had fetal heart activity noted on admission to $\mathrm{KCH}$, suggesting that most of the stillbirths occurred prior to arrival at this tertiary hospital, highlighting the potential delays in referral of these high-risk patients.
A number of studies have been conducted in Sub-Saharan Africa to evaluate the prevalence and causes of stillbirth. These studies have also used similar methodology to our study, with some studies also comparing the differences in risk factors between fresh and macerated stillbirths. A related prospective observational study performed in Nigeria recruited 158 women who delivered stillbirths over a 16-month period. ${ }^{17}$ Similar to our study, this study found that the major causes of stillbirths were placental abruption (17.7\%), hypertensive disorders of pregnancy $(12.7 \%)$, and maternal HIV infection $(10.7 \%) .{ }^{17}$ However, their stillbirth rate of 40.5 per 1,000 total births was about half that of our study, and unlike our study, uterine rupture was not a major cause of stillbirth. In a cross-sectional evaluation of 937 cases of stillbirths over 10 years in Nigeria, the major causes of stillbirths were hypertensive disorders of pregnancy $(18.9 \%)$, prolonged/obstructed labor $(13.6 \%)$, anemia in pregnancy $(12.2 \%)$, placental abruption $(9.3 \%)$, and uterine rupture $(8.8 \%) .{ }^{18}$ Similar to our study, $20 \%$ of the stillbirths in this study were unexplained.

In this study, we did not find any evidence of association between types of stillbirth amongst parturient and hypertension or diabetes. These results are not consistent with the findings from a retrospective cohort study on birth outcomes of about 100,000 women attending 
antenatal care and delivering in Lusaka, Zambia. In the Zambian study, hypertension, untreated syphilis, and diabetes were associated with macerated stillbirth but not with fresh stillbirth. ${ }^{19}$ However, our failure to find association between type of stillbirth and hypertension or diabetes may be due to the small number of stillbirths we had compared to the 2,109 stillbirths included in the Zambian study. In this study, only one woman was diagnosed with diabetes (who did have a macerated stillbirth). Otherwise, both the Zambian study and our study found that cesarean delivery and assisted vaginal deliveries were significantly associated with fresh stillbirths.

Our finding that a large number of stillbirths are unexplained stillbirths is consistent with what has been reported in other studies. A systematic review published in 2014 found that the rate of unexplained stillbirth in low to middle income countries ranged between 3.8$57.4 \%,{ }^{20}$ and another systematic review performed in 2017 showed that unexplained stillbirths had pooled estimates ranging from 31.2 to $43.7 \% .{ }^{8}$ However, most of the unexplained stillbirths in this study were macerated. This high rate of unexplained stillbirths among macerated stillbirths is similar to the findings from a study done in Ghana. In the Ghana study, about a quarter of the stillbirths were unexplained; of which about $16 \%$ were macerated stillbirth and $9 \%$ were fresh stillbirth. ${ }^{21}$

The rate of uterine rupture in our study was higher than in the studies noted above as well as other studies in the region. ${ }^{22}$ High rates of uterine rupture in our study, especially involving women with a prior cesarean, may be a reflection of poor intrapartum care of women with prior cesareans. There is therefore a need to review the outcomes of women undergoing $\mathrm{VBAC}$ at $\mathrm{KCH}$ and the referring centers to see whether prerequisites of attempting $\mathrm{VBAC}^{23}$ are being met and whether it is safe to perform a trial of labor after cesarean section in our setting, given that continuous fetal monitoring is rarely available in Malawi, even in the tertiary hospitals.

Malaria prevalence among women who delivered stillbirths in this study was lower than the expected among pregnant women. ${ }^{24}$ The low malaria prevalence can be due to seasonal variation in malaria prevalence in this region. The study period recruitment period of 6 months may have resulted in missing of risk factors for stillbirth that have seasonal variation during the year, such as malaria, which is more prevalent during the rainy season (November through April). In addition, as mentioned previously, the prevalence of stillbirth at a tertiary referral hospital like ours is likely much higher than those at other health facilities and may therefore not be generalizable.

One of the major limitation of this study was the lack of comparison with women who delivered livebirths. This would have allowed for comparison of risk factors between the women who delivered stillbirths and those that delivered livebirths. Our study did not include laboratory testing useful in identifying causes of stillbirths; these include placental pathology and fetal autopsy followed by genetic testing and testing for antiphospholipid antibodies..$^{25}$ Over a third of the patients with macerated stillbirth had unexplained stillbirth, indicating the need to perform further advanced investigations on this particular group of patients. One reason for the high unexplained stillbirths in this study is, due to limited resources, we did not use any of the structured systems to evaluate the possible causes of death (COD) for stillbirth. ${ }^{26}$ The probable causes of stillbirth in this study were however adapted from the Baird-Pattinson's classification system which mainly relies on clinical assessment of the health personnel not on post-mortem..$^{27}$ Fetal autopsy is said to be the adequate diagnostic procedure for information on the cause of deaths ${ }^{28}$ and this was not done in our study.
This study nevertheless provides data that can be useful in the training of healthcare providers in prevention of stillbirth. The study also provides a basis for future interventional studies for targeted screening and management of the causative conditions.

\section{Conclusion}

Stillbirths remain a huge problem in resource-limited settings like Malawi. Poor referral systems and a lack of diagnostic tests make it difficult to ascertain the causes of some of these stillbirths. There is need for more studies that can systematically investigate the factors associated with stillbirths in order to provide information on the strategies and interventions that need to be prioritized to reduce the high stillbirth rates.

\section{Acknowledgments}

Authors would like to thank all the staff at KCH EMMW for their contribution to the study data collection process.

\section{Authors' contribution}

FS, JHT, GC and BM contributed to the design of the study and study implementation. FS, JHT, MC, GC and BM drafted the manuscript. All authors have read and approved the final manuscript.

\section{Funding}

This study was funded by Bill \& Melinda Gates Foundation (OPP \#1090837) grant.

\section{Conflicts of interest}

No conflicts of interest reported.

\section{References}

1. Lawn JE, Blencowe H, Waiswa P, et al. Stillbirths: rates, risk factors, and acceleration towards 2030. Lancet. 2016;387(10018):587-603.

2. Lawn JE, Gravett MG, Nunes TM, et al. Global report on preterm birth and stillbirth (1 of 7): definitions, description of the burden and opportunities to improve data. BMC Pregnancy and Childbirth. 2010;10(1):S1.

3. Blencowe H, Cousens S, Jassir FB, et al. National, regional, and worldwide estimates of stillbirth rates in 2015, with trends from 2000: a systematic analysis. The Lancet Global Health. 2016;4(2):e98-108.

4. Samuelsson M, Rådestad I, Segesten K. A waste of life: fathers' experience of losing a child before birth. Birth. 2001;28(2):124-30.

5. Flenady V, Boyle F, Koopmans L, et al. Meeting the needs of parents after a stillbirth or neonatal death. BJOG. 2014;121 Suppl 4:137-140.

6. Trulsson O, Rådestad I. The silent child--mothers' experiences before, during, and after stillbirth. Birth. 2004;31(3):189-195.

7. World Health Organization. Every newborn: an action plan to end preventable deaths. World Health Organization; 2014. 55 p.

8. Reinebrant HE, Leisher SH, Coory M, et al. Making stillbirths visible: a systematic review of globally reported causes of stillbirth. BJOG. 2018;125(2):212-224.

9. Goldenberg RL, McClure EM, Bann CM. The relationship of intrapartum and antepartum stillbirth rates to measures of obstetric care in developed and developing countries. Acta Obstet Gynecol Scand. 2007;86(11):13031309 .

10. Feresu SA, Harlow SD, Welch K, et al. Incidence of and socio-demographic risk factors for stillbirth, preterm birth and low birthweight among Zimbabwean women. Paediatr Perinat Epidemiol. 2004;18(2):154-163. 
11. Shankar M, Navti O, Amu O, et al. Assessment of stillbirth risk and associated risk factors in a tertiary hospital. J Obstet Gynaecol. 2002;22(1):34-38.

12. Getahun D, Ananth CV, Kinzler WL. Risk factors for antepartum and intrapartum stillbirth: a population-based study. Am J Obstet Gynecol. 2007;196(6):499-507.

13. World Population Review. Lilongwe Population 2021 (Demographics, Maps, Graphs). 2021.

14. World Health Organization. ICD-10: international statistical classification of diseases and related health problems : tenth revision. 2021.

15. World Health Organization. Making every baby count. 2021.

16. Lawn JE, Kerber K, Enweronu-Laryea C, et al. 3.6 million neonatal deaths-what is progressing and what is not? Semin Perinatol. 2010;34(6):371386.

17. Mutihir JT, Eka PO. Stillbirths at the Jos University Teaching Hospital: incidence, risk, and etiological factors. Niger J Clin Pract. 2011;14(1):1418.

18. Njoku CO, Emechebe CI, Eyong EM, et al. Prevalence and risk factors for stillbirths in a tertiary hospital in Niger Delta area of Nigeria: a ten year review. International Journal of Medicine and Biomedical Research. 2016;5(3).

19. Stringer EM, Vwalika B, Killam WP, et al. Determinants of stillbirth in Zambia. Obstet Gynecol. 2011;117(5):1151-1159.
20. Aminu M, Unkels R, Mdegela M, et al. Causes of and factors associated with stillbirth in low- and middle-income countries: a systematic literature review. BJOG. 2014;121 Suppl 4:141-153.

21. Alhassan A, Ayikai LA, Alidu H, et al. Stillbirths and associated factors in a peri-urban District in Ghana. Journal of Medical and Biomedical Sciences. 2016;5(1):23-31.

22. Madhi SA, Briner $\mathrm{C}$, Maswime $\mathrm{S}$, et al. Causes of stillbirths among women from South Africa: a prospective, observational study. The Lancet Global Health. 2019;7(4):e503-512.

23. Birth after previous caesarean birth. RCOG Green-top Guideline No. 45 (2015).

24. Steve M Taylor, Feiko O ter Kuile. Stillbirths: the hidden burden of malaria in pregnancy. The Lancet Global Health. 2017.

25. Page JM, Christiansen-Lindquist L, Thorsten V, et al. Diagnostic tests for evaluation of stillbirth: results from the stillbirth collaborative research network. Obstet Gynecol. 2017;129(4):699-706.

26. Flenady V, Frøen JF, Pinar H, et al. An evaluation of classification systems for stillbirth. BMC Pregnancy Childbirth. 2009;9(1):24.

27. Pattinson RC, De Jong G, Theron GB. Primary causes of total perinatally related wastage at Tygerberg Hospital. South African Medical Journal. 1989.

28. ACOG Practice Bulletin 2020. Management of stillbirth. 2021. 